\title{
Microstructure and patterns of coherent radiation
}

\author{
J. G. Ables
}

Div. of Radiophysics, CSIRO, PO Box 76, Epping 2121, Australia

\section{McConnell}

Autralia Telescope National Facility, CSIRO, Locked Bag 194, Narrabri 2390, Australia

\begin{abstract}
We present data which indicates that micropulses from the bright millisecond pulsar PSR J0437-4715 are observed at equally-spaced preferred phases within the pulse window. We find the pattern similar to that expected if each micropulse is radiated from a source with a coherence scale of $\sim 100 \mathrm{~m}$.
\end{abstract}

\section{Introduction}

An important source of information on pulsar emission mechanisms comes from the study of individual pulses. In particular, observations with high timeresolution (e.g. by Hankins, 1971) have revealed structures, the so-called "micropulses", in pulsar signals down to $1 \mu$ s and less. These observations are possible only on the brightest pulsars. In general, the millisecond pulsars are too weak (both intrinsically and because of the great distance to the globular cluster pulsars) for single pulse studies. An exception is the close southern pulsar PSR J0437-4715. We have studied single pulses from this object and have measured the mean phase distribution of its micropulses.

\section{Observations}

We observed PSR J0437-4715 from the Ooty Radio Telescope in India at a frequency of $327 \mathrm{MHz}$. This Telescope has an area of $8000 \mathrm{~m}^{2}$. The single polarization signals were analyzed by a digital spectrometer (1024 channels with $\sim 10 \mathrm{kHz}$ BW / channel) which also provided on-line post-detection dedispersion and summing of all channels (McConnell et al. 1996). The sampling interval of the output 16-bit sample stream was $102.4 \mu \mathrm{s}$, which was derived from the observatory $\mathrm{Rb}$ frequency standard.

PSR J0437-4715 is one of the closest known pulsars and appears relatively bright with a mean flux at this frequency of $>1 \mathrm{Jy}$. In addition, interstellar scintillation leads to brightness enhancements of 10 times or more for a few per cent of the time. During these these favourable times, observations of single pulses were possible with a very high signal-to-noise ratio. Over an 18 day 


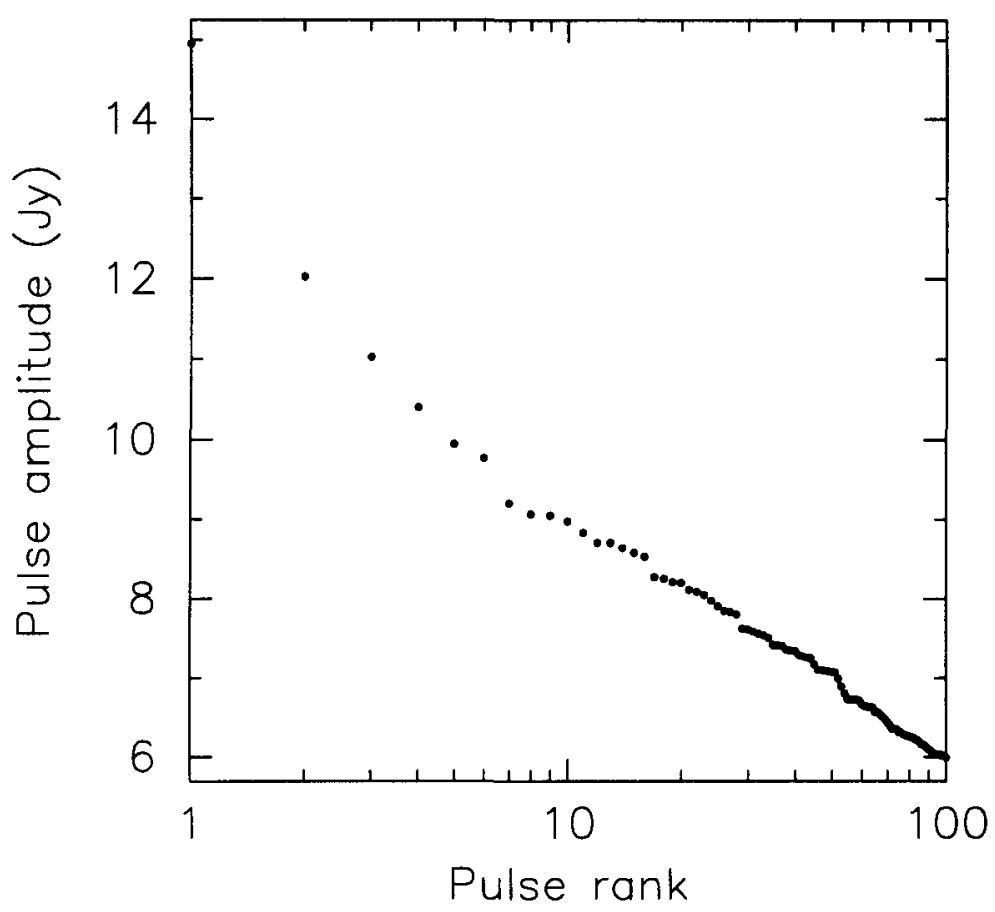

Figure 1. Rank plot of amplitudes of the 100 strongest spikes in a record made at 1995 March 7, 16:14 UT. Steps appear near ranks 6, 16,28 and higher.

period in 1995 March, we recorded 44 datasets each of $\sim 70$ s duration during the periods of high apparent brightness.

\section{Results}

The most striking feature of the single-pulse data is that many of the pulses have a very strong ( $\gg$ mean peak flux), narrow "spike" of emission at a time near the peak of the mean pulse profile. Rarely are two, and even more rarely, three, such spikes seen in the same pulse period. The widths of the spikes are unresolved at our sample rate and so the true widths are $\leq 100 \mu \mathrm{s}$. Broadening from interstellar scattering is expected to be low $(\sim<=1 \mu \mathrm{s})$ (Lang, 1980). On the other hand, every pulse displays something like the mean flux profile at a much lower level and somewhat obscured by noise. Thus, the mean profile is not simply the long-term average of the spikes which are tightly clustered about the positions of the main peaks.

Because our data are bandlimited and the samples meet the Nyquist sampling criterion, we may use the Nyquist reconstruction theorem to recover the original continuous function (pulsar signal plus noise) that existed before sampling (Bracewell 1965). The high signal-to-noise ratio at the spike peaks allows 


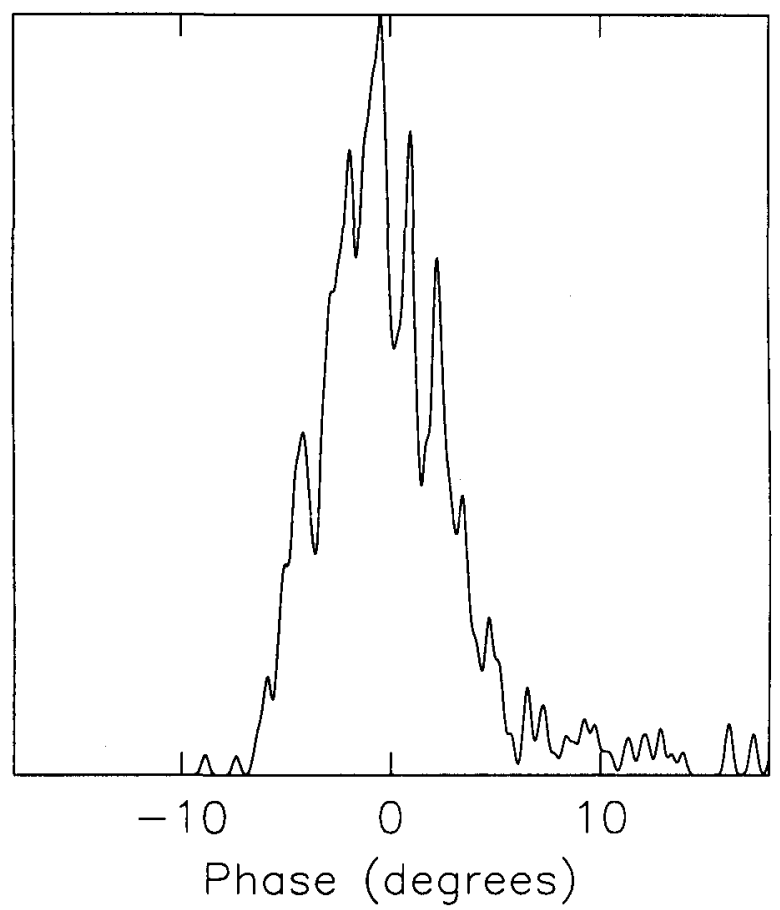

Figure 2. Profile formed by the smoothed arrival time distribution of the strongest 500 spikes (from the data set of Figure 1), weighted by their amplitudes.

us to determine the time (centre) of the spikes from the reconstructed continuous function to an uncertainty (one standard deviation) of $\sim 5 \mu$ s, only $\sim 1 / 20$ of $t_{s}$.

By means of a computed ephemeris for the pulsar (using the timing parameters of Bell et al. 1995) the arrival times were converted to pulsar rotational phases. Thus all information about the spikes was reduced to a list of pulsar phases and associated spike amplitudes. Because the spikes are unresolved there is no information about their shapes.

Analysis of the distribution of spike amplitudes and phases revealed some interesting features: spikes occur at evenly spaced, preferred phases within the pulse window; and the amplitude of brighter spikes also appears quantized (Figure 1).

The profile formed by the smoothed time of arrival distribution of the spike component of the radiation shows enhancements at the preferred phases of spike ocurrence. Figure 2 shows such a profile which exhibits about 8 evenly spaced sub-peaks. Note that the overall width of the spike profile is about 10 degrees of longitude, considerably narrower than the central component of the integrated profile. 


\section{Discussion}

The analysis described above has been performed for all 44 datasets recorded at Ooty. Patterns similar to that of Figure 2 are evident in spike profiles from all data sets. We find the patterns strongly reminescent of "fringes" observed in interference phenomena. If they are diffraction fringes, the associated spatial scale for this wavelength is $\sim 100 \mathrm{~m}$.

\section{References}

Bell, J. F., Bailes, M., Manchester, R. N., Weisberg, J. M. \& Lyne, A. G. 1995, ApJ, 440, L81

Bracewell, R. 1965, "The Fourier Transform and its Applications", McGraw-Hill, New York.

Hankins, T. H. 1971, ApJ, 169, 487

Lang, K. R. 1980, "Astrophysical Formulae", Springer-Verlag, Germany.

McConnell, D., Ables, J. G., Bailes, M. \& Erickson, W. C. 1996, MNRAS, (In Press) 